\title{
Improving risk equalization using information on physiotherapy diagnoses
}

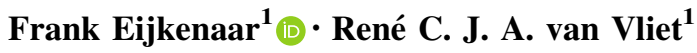

Received: 12 October 2016/ Accepted: 24 January 2017/Published online: 9 February 2017

(C) The Author(s) 2017. This article is published with open access at Springerlink.com

\begin{abstract}
Background Worldwide, risk-equalization (RE) models in competitive health insurance markets have evolved from simple demographic models to sophisticated models containing diagnosis and pharmacy-based indicators of health. However, these models still have important imperfections; adding information on (diagnoses of) physiotherapy treatment may further improve RE-models. Therefore, a new risk-adjuster based on physiotherapy costs in the prior year was introduced in the Dutch RE-model of 2016.

Methods Physiotherapy claims-data (2012) and administrative data on costs and risk-characteristics (2013) for $94 \%$ of the Dutch population ( $N=15.8$ million) are used to evaluate the current risk-adjuster based on physiotherapy costs and to assess the effects of replacing it by different modalities of a risk-adjuster based on physiotherapy diagnoses. Of the 89 diagnoses in the claims-data, 62 are dropped because they relate to temporary health problems. The 27 retained diagnoses are added to the Dutch model in 4 modalities: 27 separate risk-classes, 9 diagnosis-clusters based on main pathology category, 4 diagnosis-clusters based on residual costs, and the 4 clusters of modality 3 interacted with age.
\end{abstract}

Electronic supplementary material The online version of this article (doi:10.1007/s10198-017-0874-x) contains supplementary material, which is available to authorized users.

Frank Eijkenaar

eijkenaar@bmg.eur.nl

René C. J. A. van Vliet

r.vanvliet@bmg.eur.nl

1 Institute of Health Policy and Management, Erasmus University Rotterdam, Burgemeester Oudlaan 50, 3000 DR Rotterdam, The Netherlands
Results Although the cost-based risk-adjuster improves the model's predictive power and removes the average undercompensation (€919) for enrollees with physiotherapy costs in the prior year, it is outperformed by all 4 diagnosis-based modalities. Of these modalities, modality 3 is preferred based on its simplicity and comparable predictive power.

Conclusions Adding information on physiotherapy can further improve the performance of sophisticated REmodels. Regarding the Dutch model, a risk-adjuster containing 4 risk-classes for clustered diagnoses based on residual costs is the preferred modality.

Keywords Health insurance - Risk equalization . Physiotherapy diagnoses $\cdot$ Claims data

JEL Classification I1 1 I13 $\cdot$ G22

\section{Introduction}

In an increasing number of competitive health insurance markets, risk equalization (RE) is used as a mechanism that provides insurers with a compensation for each enrolled person based on expected costs and adjusted for individual risk characteristics. As a result, insurers receive a higher payment for high-risk enrollees (e.g. the elderly and chronically ill) than for low-risk enrollees (e.g. the young and healthy). In the presence of premium-rate restrictions, the goal of RE is to reduce incentives for risk selection while maintaining insurers' incentives for promoting efficiency of care [1].

Different forms of RE have been implemented in Europe [2-6] and the US [7, 8]. In the last decades, RE-models have developed from simple demographic models to 
sophisticated models using diagnosis and pharmacy-based indicators of health $[3,4,7,8]$. For example, the Dutch REmodel for curative somatic care now contains about 160 risk-classes, almost half of which can be considered direct proxies for health because they are based on diagnoses or prior utilization related to chronic illness [4]. However, even these sophisticated models have important imperfections and result in undercompensations for individuals with rare diseases, (functional) disability, multi-morbidity, and poor self-reported health. For example, the Dutch REmodel of 2015 results in a statistically significant average undercompensation of $€ 378$ for the subgroup of individuals who judge their own health as 'alright', 'poor', or 'very poor' (about $20 \%$ of the population) [9]. Undercompensations such as these provide insurers with incentives for risk selection, which is undesirable because it may reduce quality of care, efficiency of care, and solidarity between healthy and chronically ill people [10]. Even in the presence of regulations such as open enrollment, no exclusion of preexisting conditions, and standardized insurance coverage, many (subtle) forms of risk selection are possible [11].

A potentially viable option for further improving REmodels is to make use of information on (diagnoses from) treatments in primary care. Although current sophisticated RE-models make extensive use of diagnosis information, this information is typically only related to specific hospital treatments [3, 4, 7, 8]. With this in mind, several improvements have been proposed for the Dutch REmodel, one of which is a risk-adjuster based on the prior use of physiotherapy [9]. The rationale of this adjuster is threefold. First, the RE-model 2015 results in an average undercompensation of about $€ 1000$ for the subgroup of almost 400,000 adults with physiotherapy costs under the basic insurance in the prior year [12]. Second, under the Dutch basic insurance, coverage for physiotherapy for adults starts at the 21st reatment (provided there is a referral from a physician who sets the diagnosis) and is limited to specific chronic conditions (see table, supplemental digital content 1). For individuals age 17 and younger, all treatments related to these conditions are covered, in addition to a maximum of 18 treatments for other conditions. Thus, a risk-adjuster based on the use of physiotherapy could potentially reduce undercompensations for chronically ill people. Finally, medical experts indicated that undercompensated groups of people with rare genetic diseases often make use of physiotherapy.

Therefore, a dummy-variable for yes/no physiotherapy costs in the prior year was added to the Dutch RE-model 2016 under the name 'physiotherapy utilization groups' (PUGs). For practical reasons (e.g. the difference in coverage), the PUGs only apply to adults. Although the PUGs removed the undercompensation for the subgroup with physiotherapy costs in the prior year, this risk-adjuster has several disadvantages [12]. First, since it is based on prior costs, it is 'endogenous' and reduces insurers' incentives for promoting efficiency of care. Second, in the prior year's costdata it is not possible to distinguish between 'permanent' chronic conditions for which coverage exists regardless of the duration of the treatment, and 'temporary' conditions covered for a limited treatment duration only (see table, supplemental digital content 1). In RE, however, risk-adjusters ideally relate only to chronic conditions (not to temporary health problems) because the aim is to compensate insurers for predictable cost variation. Third, as the riskadjuster is only based on yes/no costs in the prior year, it does not take the severity of the underlying diagnosis into account. Finally, the specific characteristics of the prior year's cost data preclude inclusion of children in the risk-adjuster.

These problems may be overcome by using information on diagnoses of physiotherapy treatment (instead of on prior costs) [12]. The resulting adjuster would be a more direct proxy for health and similar to the diagnosis-based cost groups (DCGs) in the Dutch RE-model, in which enrollees are classified into risk-classes using diagnoses from hospital treatments [13, 14]. At the time the PUGs were developed, data on physiotherapy diagnoses were not available for research purposes. However, at the end of 2015, when a new research cycle for improving the REmodel of 2016 started, these data came available.

In this paper, using physiotherapy claims data of 2012 and administrative data on costs and risk characteristics of 2013 for (almost) the entire Dutch population, we evaluate the PUGs and assess the effects of replacing the PUGs by different modalities of an alternative risk-adjuster labeled 'physiotherapy-diagnosis groups' (PDGs). Specifically, our goal is threefold: (1) to study the possibilities of improving $\mathrm{RE}$ using information on (the use of) physiotherapy (2) to develop different modalities of a risk-adjuster based on physiotherapy diagnoses and (3) to assess the effects of replacing the PUGs by these modalities in terms of key criteria for adequate risk-adjusters, including predictive power, validity, and stability [1].

\section{The Dutch basic health insurance system}

Since 2006, Dutch residents have been obliged to purchase basic health insurance coverage for a standardized benefits package. In 2016, this basic insurance scheme comprises about $70 \%$ of total healthcare spending and is characterized by a form of managed competition in which insurers compete on price and quality and enrollees can switch annually among insurers. The scheme comprises three main financial streams: a community-rated premium from adult enrollees to insurers, an income-related contribution from enrollees to the RE-fund (a national account from 
which the RE-payments to insurers are financed), and REpayments between insurers and the RE-fund. The REpayments are based on the expected costs of insurers' enrollees in year $t$, which are based on the coefficients of an OLS-regression of costs on risk characteristics using data from $t$-3 (see table, supplemental digital content 2, for the coefficients of the Dutch RE-model of 2016 with and without the PUGs).

The Dutch RE-model 2016 contains the following ten risk-adjusters: age/gender (40 risk-classes), region (10 riskclasses based on clusters of zip-codes), socioeconomic status (17 risk-classes based mainly on income, interacted with age), source of income (24 risk-classes for self-employed, students, higher-educated, and people with social security benefits; interacted with age), pharmacy-based cost groups (PCGs, 30 risk-classes based on prior use of medication prescribed for chronic illnesses), DCGs (15 risk-classes based on diagnoses from hospital treatments in the prior year), durable medical equipment cost groups (DMECGs, 4 risk-classes based on prior use of durable medical equipment), multi-year high cost groups (MYHCGs, 6 risk-classes based on high costs in the prior 3 years), PUGs (1 riskclass), and 4 interaction risk-classes. In this model, the PCGs, DCGs, DMECGs and MYHCGs can be considered as 'morbidity-based' and thus as direct proxies for health.

In addition, the model contains 2 risk-adjusters based on the costs of home care and of geriatric revalidation in the prior year. However, both adjusters are excluded here because (1) the Dutch Minister of Health aims to abolish these 'endogenous' risk-adjusters ultimately by the end of 2018 [15] and (2) these adjusters may have confounding effects on the results.

\section{Methods}

Two main datasets are used for this study. First, we use a dataset containing all basic health insurance claims ( $N=14.4$ million) for physiotherapy treatments provided to about 700,000 patients in 2012. Importantly, the claims contain a diagnosis-code based on a classification system that enables the physiotherapist to register the body-location and the pathology of the diagnosis using a 4-digit coding. Digits 3 and 4 refer to the pathology, with digit 3 being the main category (e.g. neurological condition) and digit 4 the subcategory (e.g. paraplegia). For this study, only digits 3 and 4 (henceforth: diagnosis) are relevant because for a given pathology, it is highly unlikely that a difference in body-location involves a difference in health. In total, 89 different diagnoses can be discerned in the claims data.

The second dataset contains administrative, individuallevel data on costs and risk characteristics in 2013 for the entire Dutch population ( $N=16.9$ million). This dataset was actually used for calculating the RE-payments for Dutch insurers for 2016, and came from various administrative sources. Costs comprise the total costs for curative somatic care under the basic health insurance of 2016 (cost-level 2013). As is customary in the Dutch RE-model, costs of enrollees were annualized and weighted for each enrollee for the duration of the insurance contract in 2013.

Given these datasets, we used a 7-step procedure to address the research objectives:

1. Delete unusable/incorrect claims. We deleted physiotherapy claims with an unknown diagnosis-code and/or a negative claim-amount. In addition, we deleted clearly invalid claims (e.g. child physiotherapy claims submitted for adults) and all claims pertaining to the enrollees of 3 insurers because these insurers submitted incomplete or no claims. As a result of these selections, the number of patients with a physiotherapy diagnosis reduced to approximately 675,000 .

2. Merge the cleaned claims data with the administrative data. Using a unique (anonymous) identification key, we merged the 2012 claims data with the 2013 administrative data on the individual enrollee-level. As a result of the first step, the number of enrollees in the administrative data reduced from 16.9 million to 15.8 million.

3. Estimate the Dutch RE-model 2016 by OLS using 2013-costs as the dependent variable and the above mentioned risk-adjusters excluding the PUGs as the explanatory variables, and calculate the predicted costs for each enrollee (see table, supplemental digital content 2, for the coefficients of this model).

4. Calculate the mean (residual) costs for each of the 89 diagnoses (see table, supplemental digital content 3 ). Mean residual costs are calculated by subtracting for each enrollee the predicted costs from the actual costs and averaging the result to the diagnosis-level.

5. Delete diagnoses related to temporary health problems. Sixty-two diagnoses related to conditions not covered for adults (e.g. respiratory infections) and/or to conditions with limited treatment duration (e.g. fractures) were dropped. These diagnoses have a relatively low prevalence and/or low residual costs (see table, supplemental digital content 3), implying limited predictive power compared to the 27 retained diagnoses. In this step, we were assisted by a team of medical professionals, experts from health insurance companies, and experts from the Dutch Ministry of Health.

6. Construct 4 PDG-modalities based on the retained 27 diagnoses: (1) the 27 diagnoses as separate risk-classes (2) the diagnoses clustered in 9 risk-classes based on main pathology category (3) the diagnoses clustered in 
4 risk-classes based on residual costs, and (4) the 4 risk-classes from modality 3 interacted with age (0-17, 18-64, 65+). For modality 3 , diagnoses were sorted on descending residual costs, in such a way that patients with multiple diagnoses are classified in the applicable diagnosis with the highest residual costs. Using Ward's hierarchical clustering method [16], diagnoses were then clustered into 4 mutually exclusive groups based on homogeneity in residual costs, taking into account the diagnoses' standard deviation and prevalence and in such a way that the differences among the diagnosisclusters in mean residual costs is maximized. The rationale for modality 4 is that research shows that age may play a role when predicting healthcare costs using information on physiotherapy utilization [12].

7. Estimate (by OLS) 5 additional versions of the Dutch RE-model of 2016 and compare the results. These versions are the RE-model 2016 with the current PUGs and the RE-model 2016 with the PUGs replaced by 1 of the 4 PDG-modalities.

Assisted by a team of medical professionals and experts from health insurance companies, we assessed the validity of the 2012 claims data using (1) administrative data on individual-level costs for different types of primary care (including physiotherapy) for 2012 and 2013, and (2) physiotherapy claims data of 2013. Based on additional analyses on these data (see figures, supplemental digital content 4 ), the validity could be confirmed and did not lead to any further modifications or selections.

\section{Results}

\section{Descriptive statistics}

Table 1 shows descriptive statistics for all enrollees, for enrollees with physiotherapy costs in 2012, and for three groups of enrollees with a physiotherapy diagnosis in 2012. For (adult) enrollees with physiotherapy costs in 2012, total costs are on average $€ 908$ higher than for enrollees (of all ages) with at least 1 of the 27 diagnoses in 2012. However, residual costs are $€ 434$ higher in the latter group, which is particularly notable in view of the $€ 238$ lower residual costs for enrollees (of all ages) with at least 1 of the 89 original diagnoses as compared to enrollees with physiotherapy costs in 2012 .

The bottom row of Table 1 shows that almost $52 \%$ of enrollees with the 27 diagnoses are classified into one or more risk-classes of the current 'morbidity-based' riskadjusters. This implies that any version of a risk-adjuster based on the 27 diagnoses will show considerable overlap with these risk-adjusters. This overlap, however, is substantially less compared to the current PUGs (see also table, supplemental digital content 5).

As a result of dropping 62 diagnoses, the (weighted) number of patients with at least 1 diagnosis decreased from 664,139 to 286,342 . As shown in the last two columns of Table 1, mean residual costs for the dropped diagnoses are almost eight times lower than for the 27 retained diagnoses. Thus, the dropped diagnoses either have a low prevalence,

Table 1 Descriptive statistics for the year 2013

\begin{tabular}{|c|c|c|c|c|c|}
\hline & $\begin{array}{l}\text { All enrollees in } \\
\text { merged dataset }\end{array}$ & $\begin{array}{l}\text { Enrollees with physiotherapy } \\
\text { costs in } 2012 \text { (i.e. PUG >0) }\end{array}$ & $\begin{array}{l}\text { Enrollees with } \\
\geq 1 \text { of } 89 \text { original } \\
\text { diagnoses in } \\
2012\end{array}$ & $\begin{array}{l}\text { Enrollees with } \\
\geq 1 \text { of } 62 \text { dropped } \\
\text { diagnoses in } 2012\end{array}$ & $\begin{array}{l}\text { Enrollees with } \\
\geq 1 \text { of } 27 \text { selected } \\
\text { diagnoses in } 2012\end{array}$ \\
\hline Included age groups & All ages & Ages $18+$ only & All ages & All ages & All ages \\
\hline 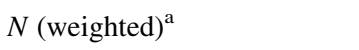 & $15,523,485$ & 312,282 & 664,139 & 377,797 & 286,342 \\
\hline Costs in $€[$ mean (st. dev.)] & $2162[7927]$ & $8836[17,065]$ & $5155[14,099]$ & $3054[8702]$ & $7928[18,645]$ \\
\hline $\begin{array}{l}\text { Residual in } €[\text { mean } \\
\text { (st. dev.) }]^{\mathrm{b}}\end{array}$ & $0[6850]$ & $919[14,496]$ & $681[11,708]$ & $172[7312]$ & $1353[15,704]$ \\
\hline Age (mean) & 40.8 & 61.0 & 34.7 & 33.9 & 35.9 \\
\hline $\operatorname{Men}(\%)$ & 49.3 & 37.2 & 45.4 & 42.6 & 49.1 \\
\hline Classified in a PCG (\%) & 19.6 & 55.6 & 28.9 & 21.8 & 38.4 \\
\hline Classified in a DCG (\%) & 9.4 & 41.8 & 22.2 & 17.3 & 28.7 \\
\hline Classified in a DMECG $(\%)$ & 0.9 & 5.4 & 2.8 & 1.5 & 4.5 \\
\hline Classified in a MYHCG (\%) & 5.9 & 47.7 & 24.7 & 15.6 & 36.6 \\
\hline $\begin{array}{l}\text { Classified in a PCG, DCG, } \\
\text { DMECG, or MYHCG }(\%)\end{array}$ & 24.6 & 73.9 & 40.6 & 32.1 & 51.8 \\
\hline
\end{tabular}

a $N=$ the number of enrollees weighted by the duration of enrollment in 2013

b Residual costs based on Dutch RE-model 2016 without the PUGs as a risk adjuster. Because the model is estimated by OLS, the average residual for all enrollees equals 0 (by definition) 
low residual costs, or both, confirming the adequacy of basing the PDG-modalities on the 27 diagnoses.

\section{Stability over time}

Ideally, the prevalence of a risk-adjuster is stable at the individual insurer-level; large fluctuations over time may indicate problems or differences in claims registration among insurers [1]. Figure 1 shows the prevalence of the 27 diagnoses and the PUGs, respectively, at the level of the 23 insurers, for 2 years. Figure 1 indicates that a risk-adjuster based on the 27 diagnoses will be more stable at the insurer-level than the PUGs (based on physiotherapy costs). In panel a, the mean absolute change from 2012 to 2013 is 0.28 per 1000 patients. In panel b, this is 1.17 , while the overall prevalence is only $9 \%$ higher compared to panel A.

In an additional analysis, we examined the changes in prevalence at the diagnosis-level (see figure, supplemental digital content 6 ). The changes are very small, especially in comparison to the common year-to-year changes in the prevalence of the current PCGs and DCGs [17].

\section{PDG-modalities}

Table 2 presents information for the PUGs and for 3 of the 4 PDG-modalities (for clarity of presentation, modality 4 is excluded in this table). For modality 1 and 2, summation of the (weighted) number of patients yields 289,375, about 3000 more than the 286,342 patients in Table 1 . This means that only $1 \%$ of enrollees have multiple diagnoses, so classifying enrollees in the diagnosis with the highest residual costs, as done in all modalities when estimating the coefficients, will have very limited consequences for the predictive power.

In modality 1 , residual costs vary from $€ 352$ to $€ 22,730$. The estimated coefficients are on average approximately $€ 250$ higher than the residual costs, which means that these risk-classes take over some of the predictive power of the other risk-adjusters in the model. The variation in coefficients for modality 2 is much smaller as compared to modality 1 .

The (residual) costs and thus the coefficients of the clusters of modality 3 sharply increase as the cluster number goes up. An additional analysis shows that the clusters explain $94 \%$ of the variance in residual costs
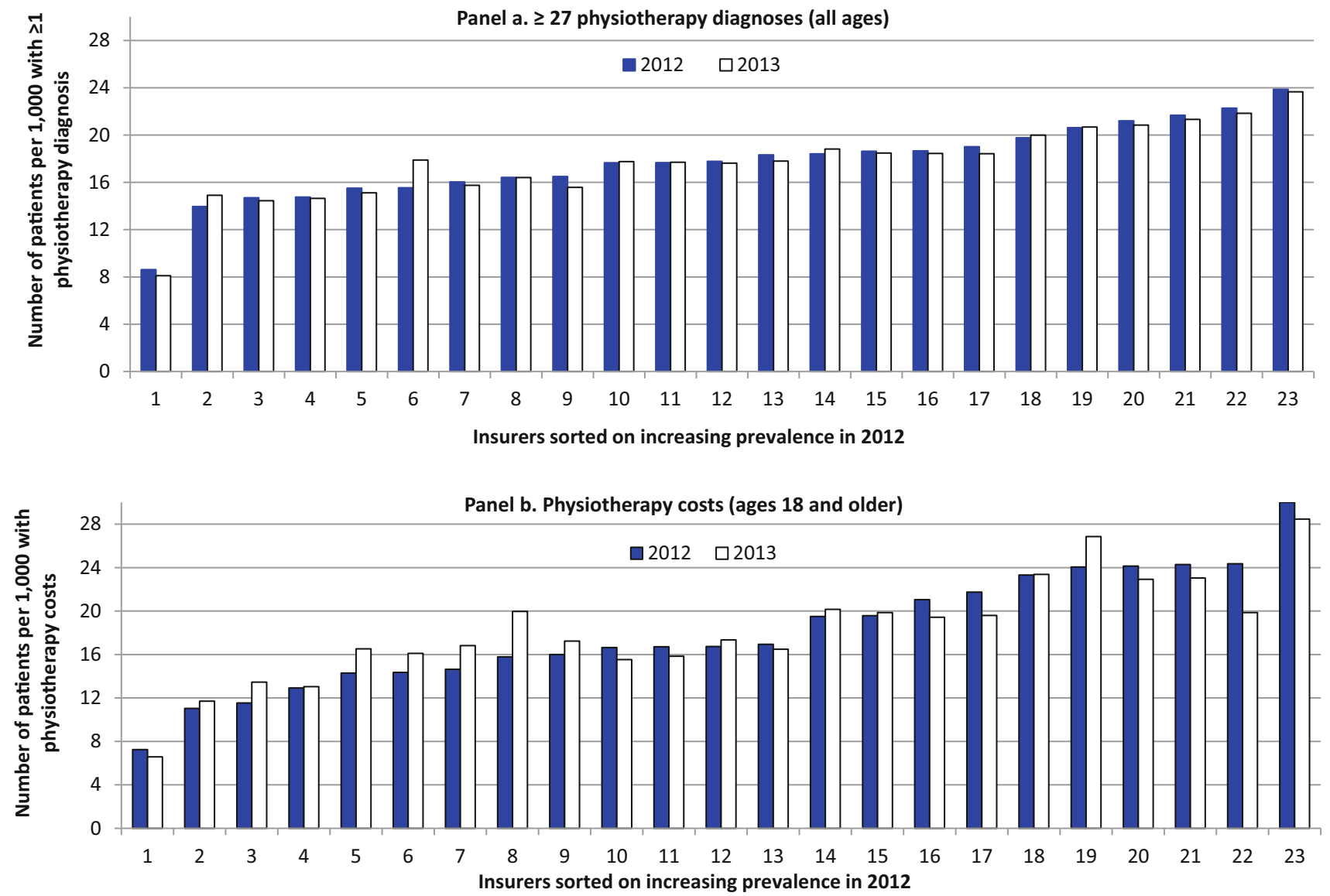

Fig. 1 Number of patients per 1000 with at least one of 27 physiotherapy diagnoses (panel $a$ ) or with physiotherapy costs (panel $b$ ) for 23 riskbearing insurers, for 2 years. In both panels, the numbers for the year 2012 are adjusted for the overall change in prevalence from 2012 to 2013 
Table 2 Key statistics for three PDG-modalities for the year 2013 (costs and coefficients in $€$ )

\begin{tabular}{|c|c|c|c|c|c|}
\hline Code/cluster & Description & $N^{\mathrm{a}}$ & $\begin{array}{l}\text { Mean } \\
\text { costs }\end{array}$ & $\begin{array}{l}\text { Mean residual } \\
\text { costs }^{\text {b }}\end{array}$ & $\begin{array}{l}\text { Estimated } \\
\text { coefficient }^{\mathrm{c}}\end{array}$ \\
\hline \multicolumn{6}{|l|}{ PUG } \\
\hline & Physiotherapy costs in 2012 & 312,282 & 8836 & 919 & 1008 \\
\hline \multicolumn{6}{|c|}{ PDG-modality 1} \\
\hline Code 00 & Amputation & 2536 & 15,603 & 1737 & 1971 \\
\hline Code 10 & Aseptic bone necrosis & 375 & 1377 & $354^{\mathrm{ns}}$ & 423 \\
\hline Code 11 & Disorders of the vertebral column/pelvis & 19,344 & 2333 & 355 & 435 \\
\hline Code 12 & Congenital disorders of the skeleton & 5895 & 5250 & 1235 & 1334 \\
\hline Code 13 & Ossification disorder & 1107 & 4311 & 473 & 567 \\
\hline Code 14 & Inflammation of/tumors in the skeleton & 676 & 16,476 & 5948 & 6125 \\
\hline Code 28 & Sudeck’ a(dys)trophy & 4692 & 8351 & 1043 & 1173 \\
\hline Code 39 & Status after burn wounds & 149 & 7063 & 1732 & 1889 \\
\hline Code 46 & Disorders of the lymphatic system/edema & 21,578 & 9120 & 399 & 471 \\
\hline Code 51 & Congenital disorders of respiratory system & 566 & 35,374 & 11,253 & 12049 \\
\hline Code 54 & Chronic obstructive pulmonary disease & 23,308 & 14,470 & 1840 & 2117 \\
\hline Code 56 & Interstitial lung disorder including sarcoïdosis & 920 & 14,792 & 3767 & 3534 \\
\hline Code 65 & Other hereditary disorders & 460 & 35,808 & 22,767 & 23,014 \\
\hline Code 69 & Tumors without surgery & 3273 & 17,060 & 1915 & 2058 \\
\hline Code 70 & Peripheral nerve disorder & 6404 & 9791 & 1819 & 1918 \\
\hline Code 71 & Cerebellar disorders & 6755 & 11,562 & 1068 & 1281 \\
\hline Code 72 & Cerebrovascular accident/central paresis & 27,049 & 13,049 & 2566 & 2773 \\
\hline Code 73 & MS/ALS/spinal muscle atrophy & 9037 & 17,831 & 3069 & 3692 \\
\hline Code 74 & Parkinson's disease/extrapyramidal disorder & 15,077 & 14,063 & 1474 & 2408 \\
\hline Code 76 & Paraplegia, including traumatic and partial & 2553 & 25,125 & 2455 & 3220 \\
\hline Code 77 & Neurotraumata & 2272 & 8166 & 1609 & 1765 \\
\hline Code 78 & Other neurological disorders & 12,171 & 15,652 & 3820 & 4089 \\
\hline Code 79 & Psychomotor retardation/development disorders & 117,681 & 2607 & 738 & 832 \\
\hline Code 94 & Other collagen disorders & 4219 & 9252 & 1800 & 1782 \\
\hline Code 95 & Scar tissue & 239 & 8642 & $1701^{\mathrm{ns}}$ & 1897 \\
\hline Code 96 & Scleroderma & 502 & 16,505 & 2078 & 2334 \\
\hline Code 99 & Other skin disorders & 537 & 8354 & $472^{\mathrm{ns}}$ & 657 \\
\hline \multicolumn{6}{|c|}{ PDG-modality 2} \\
\hline Code $0 \mathrm{x}$ & Surgery, musculoskeletal system & 2536 & 15,773 & 1820 & 1924 \\
\hline Code $1 \mathrm{x}$ & Orthopedic disorders without surgery & 27,234 & 3387 & 689 & 858 \\
\hline Code $2 \mathrm{x}$ & Surmenage, degenerative disorders, dystrophy & 4692 & 8392 & 1054 & 1151 \\
\hline Code $3 x$ & Traumatic disorders excl. surgery/neurotraumata/paraplegia & 149 & 7254 & 1896 & 1910 \\
\hline Code $4 \mathrm{x}$ & $\begin{array}{l}\text { Disorders of heart/veins/lymphatic system incl. cardio } \\
\text { surgery }\end{array}$ & 21,578 & 9146 & 386 & 469 \\
\hline Code $5 x$ & Lung disorders & 24,769 & 14,984 & 2098 & 2343 \\
\hline Code $6 x$ & $\begin{array}{l}\text { Other internal disorders excl. surgery/musculoskeletal } \\
\text { system }\end{array}$ & 3733 & 19,393 & 4432 & 4641 \\
\hline Code $7 x$ & Neurological disorders & 198,031 & 7267 & 1401 & 1654 \\
\hline Code $9 x$ & Collagen disorders and skin disorders & 5494 & 9840 & 1699 & 1709 \\
\hline \multicolumn{6}{|c|}{ PDG-modality 3} \\
\hline Cluster 1 & Included diagnoses: 10, 11, 12, 13, 28, 46, 71, 79, 99 & 175,508 & 3859 & 661 & 779 \\
\hline Cluster 2 & Included diagnoses: $0,39,54,69,70,74,77,94,95$ & 57,039 & 13,414 & 1715 & 2095 \\
\hline Cluster 3 & Included diagnoses: $14,56,72,73,76,78,96$ & 52,770 & 15,223 & 2984 & 3295 \\
\hline
\end{tabular}


Table 2 continued

\begin{tabular}{llllll}
\hline Code/cluster & Description & $N^{\mathrm{a}}$ & $\begin{array}{l}\text { Mean } \\
\text { costs }\end{array}$ & $\begin{array}{l}\text { Mean residual } \\
\text { costs }^{\mathrm{b}}\end{array}$ & $\begin{array}{l}\text { Estimated } \\
\text { coefficient }^{\mathrm{c}}\end{array}$ \\
\hline Cluster 4 & Included diagnoses: 51, 65 & 1025 & 35,571 & 16,340 & 17,056 \\
\hline
\end{tabular}

${ }^{a} N=$ the number of patients weighted by the duration of enrollment in 2013. For PDG-modality 1 and 2, enrollees may be classified in more than one diagnosis. For PDG-modality 3, enrollees are classified in the cluster containing the applicable diagnosis with the highest residual costs

b Residual costs based on the RE-model of 2016 without the PUGs as a risk-adjuster. The subscript 'ns' indicates that the residual is not statistically significant different from 0 at a significance level of $1 \%$

${ }^{c}$ Coefficient based on the RE-model of 2016 with the PUGs or with the PUGs replaced by PDG-modality 1, 2 or 3. For all PDG-modalities, enrollees are classified in the applicable diagnosis with the highest residual costs. All coefficients are statistically significant different from 0 a significance level of $1 \%$

Table 3 Fit statistics on the individual-level for five versions of the Dutch RE-model of 2016

\begin{tabular}{|c|c|c|c|}
\hline Model & $R^{2} \times 100 \%^{\mathrm{a}}$ & $\mathrm{CPM} \times 100 \%^{\mathrm{b}}$ & MAPE $^{\mathrm{c}}$ \\
\hline RE-model 2016 without PUGs & 25.33 & 27.19 & 1976.54 \\
\hline RE-model 2016 with PUGs & 25.36 & 27.28 & 1973.97 \\
\hline RE-model 2016 without PUGs + PDG-modality 1 & 25.45 & 27.32 & 1972.83 \\
\hline RE-model 2016 without PUGs + PDG-modality 2 & 25.41 & 27.28 & 1973.95 \\
\hline RE-model 2016 without PUGs + PDG-modality 3 & 25.45 & 27.33 & 1972.78 \\
\hline RE-model 2016 without PUGs + PDG-modality 4 & 25.45 & 27.33 & 1972.69 \\
\hline
\end{tabular}

among the 27 diagnoses, implying almost no information loss as a result of the clustering. Compared to modality 2 , the (residual) cost variation is much better captured, as becomes clear from the variation in coefficients.

In modality 4 , over $75 \%$ of the patients classified in the first cluster are below age 18, while the 3 other clusters consist for $95 \%$ of adult patients (data not shown). This implies that if the RE-model includes modality 1,3 or 4, the substantial residual-cost differential between children and adults with a physiotherapy diagnosis (i.e. $€ 770$ versus $€ 1936)$ is already accounted for. Within the clusters, the coefficients vary by age (see supplemental digital content 2 ), but this has limited meaning when the prevalence per risk-class is taken into account.

Finally, the fit statistics in Table 3 imply that while all PDG-modalities (slightly) outperform the PUGs in terms of predictive power, modality 3 is preferred over the other modalities in view of its equal or better fit and the lowest number of risk-classes.

\section{Discussion and conclusion}

Although RE-models in competitive health insurance markets have become increasingly sophisticated, they often still result in substantial undercompensations for specific groups of people in poor health. A potentially viable option to further improve RE-models is to make use of information on (diagnoses of) treatments in primary care instead of only hospital care (as is common in current RE-models $[3,4,7,8])$. Hence, a new risk-adjuster based on the prior use of physiotherapy ('physiotherapy utilization groups', PUGs) was introduced in the Dutch RE-model of 2016. However, this risk-adjuster has clear disadvantages since it is based on prior costs and does not take the severity of the underlying diagnosis into account.

Using physiotherapy claims data of 2012 and administrative data on costs and risk characteristics of 2013 for almost the entire Dutch population, this paper investigated the possibilities of improving RE using information on (use of) physiotherapy by (1) evaluating the current cost-based PUGs and (2) assessing the effects of replacing this risk-adjuster by 4 different modalities of an alternative risk-adjuster based on 27 physiotherapy diagnoses related to specific chronic conditions ('physiotherapy diagnosis groups', PDGs).

Although adding the PUGs removes the average undercompensation of $€ 919$ for the subgroup of enrollees with physiotherapy costs in the prior year and (slightly) improves the model's predictive power, all 4 PDGmodalities have clear advantages over the PUGs in terms of validity, stability, measurability, incentives for efficiency, predictive power, and simplicity/transparency. 
The PDG-modalities are more valid indicators of health than the PUGs because (1) they are based on diagnoses instead of on costs, (2) only diagnoses pertaining to chronic conditions are included instead of also temporary health problems, (3) they take the severity of the underlying diagnoses into account, and (4) all ages are included instead of adults only.

In addition, insurers' efficiency incentives are not reduced since the PDG-modalities are not based on prior costs. On the other hand, an inherent disadvantage of using diagnosis-based risk-adjusters is that it may provide incentives for insurers to promote classifying patients into more 'severe' diagnoses-categories than is medically necessary because these diagnoses are associated with higher payment. However, while in general such upcoding practices can be a significant problem in diagnosis-based payment schemes, it is unlikely to be very relevant in the context of this study. First, physiotherapy treatment for adults for the conditions related to the 89 analyzed diagnoses is not covered under the Dutch basic health insurance until the 21 st treatment. Although for children coverage does start at the first treatment, the estimated coefficients for the diagnoses that are most relevant for this group are relatively low (i.e. the average coefficient for these diagnoses is lower than that of the current PUGs), mitigating possible upcoding incentives. More importantly, the Dutch physiotherapy diagnosis classification system contains only 89 different and clearly distinct (pathology) categories. Of these, only 27 are relevant for the PDG-modalities, which all pertain to serious 'permanent' chronic conditions for which upcoding does not seem to be a real issue. Still, although we thus believe there to be limited potential for upcoding in this context, as with any diagnosis-based payment scheme, monitoring changes in diagnosis prevalence over time as well as periodic validation of set diagnoses (e.g. by contrasting diagnosis prevalence patterns with utilization patterns of related types of care) is advisable.

Regarding measurability, the advantage of the PDGmodalities is that no arbitrary decisions are required for determining when someone will be classified in the riskadjuster or not. In the current RE-model, enrollees are classified in a PUG $>0$ if their physiotherapy costs in the prior year are in the top-2\%. Although this threshold was introduced to eliminate the impact of administrative (data) flaws and to mitigate the reduction in efficiency incentives, it was arbitrarily determined. On the other hand, in constructing the PDG-modalities the enrollees of three insurers were necessarily dropped because of lacking or inadequate claims. However, this problem appears to have been solved in the 2013-claims data.

Regarding stability, this paper has shown that in terms of the number of patients classified, the PDGs are more stable at the insurer-level than the PUGs. In addition, the prevalence of the 27 diagnoses varies only marginally over time. Although our data did not permit analysis of the stability of coefficients, findings from the recent annual update of the RE-model confirm that the (pattern in the) coefficients of PDG-modality 3 (i.e. the 27 diagnoses clustered in 4 risk-classes based on residual costs) are indeed stable [18]: the weighted average absolute change in estimated coefficients from 2013 to 2014 equals $€ 2$.

Relative to the PUGs, all PDG-modalities (slightly) improve the model's predictive power. In turn, modality 3 outperforms the other modalities given its low number of risk-classes and comparable or better predictive power.

Finally, all PDG-modalities are arguably more transparent than the PUGs because (1) they show considerably less overlap with the 'morbidity-based' adjusters already in the model, and (2) 'diagnoses' are more salient and easier to interpret than 'costs'. Of the 4 modalities, modality 3 may be viewed as being the simplest because it consists of only 4 risk-classes. However, as a result of the clustering method these risk-classes have lost their (pathology) label and thus are not clinically recognizable, reducing the transparency. On the other hand, modality 3 is based on the same clustering method as the current DCGs [14], enhancing model consistency.

Our findings must be viewed in the light of three limitations. First, about 1 million enrollees of three insurers were necessarily dropped from the analysis due to inadequate information on physiotherapy claims of 2012. Nonetheless, analysis was possible for $94 \%$ of the Dutch population. Second, our data did not permit analysis of the stability of the coefficients of the PDG-modalities. However, findings of follow-up research indicate that the coefficients of modality 3 are indeed stable [18]. Finally, both the 2012 and the 2013 data contain claims that are actually not covered under the basic health insurance. However, these claims (which were excluded from the analysis) comprise less than $2 \%$ of the total number of claims in both years.

In conclusion, adding information on physiotherapy (diagnoses) can further improve the performance of already sophisticated RE-models. Of the different physiotherapybased risk-adjuster modalities analyzed in this paper, the modality based on clustered physiotherapy diagnoses based on residual costs is the preferred modality. Compared to a risk-adjuster based on prior physiotherapy costs, such a diagnosis-based risk-adjuster is more valid, more stable, more easily measured, more transparent, has more predictive power, and does not compromise insurers' incentives for promoting efficiency of care.

Acknowledgements The authors gratefully acknowledge the comments on previous versions of this paper by Richard van Kleef. The 
authors also thank Ellen van Rooijen for her contribution as a medical expert, the members of the supervisory committee for their comments, and the Dutch Ministry of Health, Welfare and Sports for financial support. Remaining errors are the responsibility of the authors.

\section{Compliance with ethical standards}

Funding This study was funded by the Dutch Ministry of Health, Welfare, and Sports.

Conflict of interest The authors declare that they have no conflict of interest.

Open Access This article is distributed under the terms of the Creative Commons Attribution 4.0 International License (http://crea tivecommons.org/licenses/by/4.0/), which permits unrestricted use, distribution, and reproduction in any medium, provided you give appropriate credit to the original author(s) and the source, provide a link to the Creative Commons license, and indicate if changes were made.

\section{References}

1. Van de Ven, W.P.M.M., Ellis, R.P.: Risk adjustment in competitive health insurance markets. In: Culyer, A.J., Newhouse J.P., (eds.) Handbook of Health Economics, pp. 755-845. Amsterdam (2000)

2. Schokkaert, E., Van de Voorde, C.: Health care reform in Belgium. Health Econ. 14, S25-S39 (2005)

3. Buchner, F., Goepffarth, D., Wasem, J.: The new risk adjustment formula in Germany: implementation and first experiences. Health Policy 109(3), 253-262 (2013)

4. Van Kleef, R.C., Van Vliet, R.C.J.A., Van de Ven, W.P.M.M.: Risk equalization in The Netherlands: an empirical evaluation. Expert Rev. Pharmacoecon. Outcomes Res. 13(6), 829-839 (2013)

5. Von Wyl, V., Beck, K.: Do insurers respond to risk adjustment? A long-term, nationwide analysis from Switzerland. Eur. J. Health Econ. 17(2), 171-183 (2016)

6. Shmueli, A.: On the calculation of the Israeli risk adjustment rates. Eur. J. Health Econ. 16(3), 271-277 (2015)

7. Pope, G.C., Kautter, J., Ingber, M.J., et al.: Evaluation of the CMS-HCC risk adjustment model. RTI for centers for medicare
$\&$ medicaid services, Medicare plan payment group, Division of risk adjustment and payment policy (2011)

8. Kautter, J., Pope, G.C., Ingber, M., et al.: The HHS-HCC risk adjustment model for individual and small group markets under the Affordable Care Act. Medicare Medicaid Res. Rev. 4(3), 9 (2014)

9. Van Kleef, R.C., Van Vliet, R.C.J.A., Eijkenaar, F.: Risk equalization for curative (somatic) care: what is the effect of the 2016-changes of the Dutch RE-model for subgroups of people identified via health survey information? Research report (in Dutch). Erasmus University Rotterdam, Rotterdam (2015)

10. Van de Ven, W.P.M.M., Van Kleef, R.C., Van Vliet, R.C.J.A.: Risk selection threatens quality of care for certain patients: lessons from Europe's health insurance exchanges. Health Aff. 34(10), 1713-1720 (2015)

11. Van de Ven W.P.M.M., Van Vliet, R.C.J.A., Van Kleef, R.C.: How can the regulator show evidence of (no) risk selection in health insurance markets? Conceptual framework and empirical evidence. Eur. J. Health Econ. (2016). February 2, Epub ahead of print

12. Eijkenaar, F., Van Kleef, R.C., Van Veen, S.H.C.M., et al.: Research for the Dutch risk equalization model of 2016: predictive power of use of physiotherapy for healthcare costs. Research report (in Dutch). Erasmus University Rotterdam, Rotterdam (2014)

13. Lamers, L.M.: Risk-adjusted capitation payments: developing a diagnostic cost groups classification for the Dutch situation. Health Policy 45, 15-32 (1998)

14. Van Kleef, R.C., Van Vliet, R.C.J.A., Van Rooijen, E.M.: Diagnoses-based cost groups in the Dutch risk-equalization model: the effects of including outpatient diagnoses. Health Policy 115(1), 52-59 (2014)

15. Schippers, E.I.: Reform of the healthcare system. Letter from the Minister of Health to the House of Representatives (in Dutch). 29689 (61). The Hague: House of Representatives

16. Ward, J.H.: Hierarchical grouping to optimize an objective function. J. Am. Stat. Assoc. 58(301), 236-244 (1963)

17. Eijkenaar, F., Van Kleef, R.C., Van Veen, S.H.C.M., et al.: Research for the Dutch risk equalization model of 2016: data collection and validation phase. Research report (in Dutch). Erasmus University Rotterdam, Rotterdam (2015)

18. Cattel, D., Eijkenaar, F., Van Kleef, R.C., et al.: Research for the Dutch risk equalization model of 2017: overall (stability) assessment phase. Research report (in Dutch). Erasmus University Rotterdam, Rotterdam (2016) 